Palliative Indicators Tool - to aid identification of palliative care needs. 10 hours teaching.

Results

- Hospice referrals for dementia patients increased: 2015/16 $76 ; 2016 / 17153$.

- Referrals from mental health increased: 2016/17 one referral; 2016/17 six referrals.

- Baseline audit of 24 inpatients demonstrated two were already on My Care Choices Register and mental health ward staff were unaware of this

- By May 2017 My Care Choices Register checked for every admission, seven referrals, three received end of life care on the ward.

- Issues - pain, agitation, nutrition/hydration, ethical dilemmas, falls, infection, advance care planning, carer burden.

- Other outcomes - positive educational feedback, reciprocal job shadowing (nurses), Namaste training (Stacpoole et al., 2015).

Conclusion This project innovatively enhanced collaborative working, increased referrals and established the My Care Choices Register in this setting. Dementia inpatient teams were enabled to improve skills and confidence in providing palliative care. Symptom management was addressed and hospital admissions were avoided for dying patients. Reciprocal educational needs were highlighted. Further work is being undertaken to consolidate this work, identify further dementia/ palliative care educational needs and build on the collaboration.

\section{P-99 DEMENTIA CARE IS EVERYONE'S BUSINESS}

${ }^{1}$ Helen Reeves, ${ }^{2}$ Angela Causton, ${ }^{3}$ Michael Hurt. ${ }^{1}$ St Giles Hospice, Whittington, UK; ${ }^{2}$ Pathways 4 Life, Walsall, UK; ${ }^{3}$ NHS Walsall Clinical Commissioning Group, Walsall, UK

\subsection{6/bmjspcare-2017-hospice.125}

Background Studies have found that people with dementia are often admitted to hospital unnecessarily from care homes by staff who have not received enough support to help them understand dementia and how to support them at the end of life.

Aims The presentation will cover how working collaboratively has resulted in a change across the culture of Walsall healthcare resulting in improved outcomes for patients with dementia. It will provide practical solutions to commonly found problems when looking at service improvements and provide an honest account of setting up a collaborative partnership between organisations and the challenges we encountered and the ways we overcame them.

Method Support is provided by a range of methods including observations, support sessions, forums and steering groups. Homes can access as much or as little as they want and have ongoing contact to ensure their needs are met. Collaboratively work with other services for more joined up working.

Results As a direct result of the service we now have a community dementia steering group that brings together a range of professionals including care homes, ambulance service, hospital, hospice, community professionals, commissioners to name a few. Here we focus on how we can all work collaboratively to improve care for dementia patients. The service is now recurrently funded and this in part is due to the service having a direct impact on the decreasing the number of patients with dementia being admitted to the acute sector.

Conclusion There is no quick fix to improving cross boundary and sector working, however, as proven by this service by acknowledging that we all have a part to play and that we can all positively influence patients care change does not have to cost anything other than time and the dedication of the staff involved.

\section{P-100 SYSTEMATIC REVIEW OF FACILITATORS AND CHALLENGES TO DYING AT HOME WITH DEMENTIA}

${ }^{1}$ Caroline Mogan, ${ }^{1}$ Mari Lloyd-Williams, ${ }^{2}$ Karen Harrison Dening, ${ }^{1}$ Chris Dowrick. ${ }^{1}$ Academic Palliative and Supportive Care Studies Group, Institute of Psychology Health and Society, University of Liverpool, UK; ${ }^{2}$ Dementia UK, UK

\subsection{6/bmjspcare-2017-hospice. 126}

Background Place of death is important in end of life care and it is reported that given the right support, most people would choose to die at home. A very small minority of people with dementia die at home and knowledge gaps remain on how best to support end of life care at home for people with dementia.

Aim This systematic review synthesised qualitative and quantitative studies to explore the challenges and facilitators of providing end of life care at home for people with dementia.

Methods A narrative approach was adopted to provide a comprehensive synthesis of previously published literature in the area. A systematic literature search was conducted across six electronic databases (AMED, BNI, CINAHL, EMBASE, MEDLINE, and PsychInfo) and reference lists of key journals were searched up to November 2016. Included studies were appraised for quality and data thematically synthesised.

Results Searches returned 1949 unique titles, of which seven studies met all eligibility criteria (four quantitative, three qualitative). All studies were rated for quality and six key themes identified - four facilitators and two challenges. Facilitators included 'support from health care professionals', 'informal caregiver resilience', 'medications and symptom management' and 'appropriate equipment and home adaptations'. Challenges included 'worsening of physical or mental health' and 'issues with formal services'.

Conclusions People with dementia may not always require specialist palliative care at the end of life and many clinicians, services and charities support people with dementia to die well at home. Informal caregivers also provide a significant amount of this care to people. Further research is required to establish how clinicians, statutory services, voluntary agencies and volunteers can support families to allow a greater number of people with dementia to die at home and to determine what services are required to facilitate home death for people with dementia.

\section{P-101 DEVELOPING A COMMUNITY DEMENTIA COMPANION SERVICE - EXTENDING VOLUNTEER LED HOSPICE SERVICES}

Claire Halsey, Lyn Deakin, Dawn Lawton. East Cheshire Hospice, Macclesfield, UK

10.1136/bmispcare-2017-hospice. 127 
Background The role of hospices in helping people live well with dementia is now clearly recognised (Hospice UK, 2015). Extending care into the community is a key method to build the important relationship between people with dementia, carers and hospice services. Based on this we developed a volunteer-led Community Dementia Companion Service to support service users, build carer resilience and forge their relationship with our hospice to meet future needs.

$\operatorname{Aim}(\mathbf{s})$ To develop, deliver and evaluate a Community Dementia Companion (CDC) Service led by hospice volunteers which increases social opportunities for service users and breaks for carers. To measure the service's impact on service user and carer well-being, develop a training and supervision regime for volunteers and establish sustainable service delivery.

Methods Literature and guidance on volunteering in palliative and dementia care were reviewed, national and local hospice strategic priorities informed service development as did service user and carer feedback. As a result a long term dementia companionship model using a volunteer workforce was developed which is now being trialled.

Results The CDC service is now operational and the first cohort of nine service users are starting to receive companion visits - early feedback on the service, and volunteer support model, is favourable.

Volunteer feedback: 'I look forward to my visits, being a friend to my person with dementia and giving the carer some 'me time'.

Service user feedback: 'I enjoy my walks'

Carer feedback: 'A wonderful experience for Alan and I and extremely valuable as it's ongoing, not time limited.'

Conclusions Although in its early stages the CDC example of innovation in hospice-enabled dementia care has potential to improve the lives of those with dementia and their carers, use volunteers well and increase familiarity with and timely use of other hospice services. CDC Service development funded by East Cheshire Council.

\section{P-102 HOW PRACTICE DEVELOPMENT CAN ENABLE ADMIRAL NURSES TO SUPPORT PEOPLE WITH DEMENTIA AT END OF LIFE}

Caroline Scates. Dementia UK, London, UK

\subsection{6/bmispcare-2017-hospice.128}

Background Admiral Nurses are specialist dementia nurses who use a range of psychosocial, educative and practical approaches to support families living with dementia. Providing Admiral Nurses with a full programme of professional and practice development is a strategic aim of Dementia UK, therefore identifying learning priorities at the end of life is vital.

Aim Admiral Nurses have the support of a practice development approach to their learning based on a competency framework developed by the University of Worcester (2016). Admiral Nurses have supported time away from practice, to develop skills and knowledge to support the people in their care, including end of life. This work explores how a practice development approach enables Admiral Nurses to develop their understanding, skills and knowledge of end of life issues for people with dementia.

Method A literature review was undertaken and two focussed discussions with Admiral Nurse Groups exploring end of life care challenges in practice. Feedback around gaps in practice then informs learning within future practice development sessions.

Results One example of a gap in practice identified was the cessation of the Liverpool Care Pathway. Davies et al. (2015) developed a toolkit of heuristics for practitioners when caring for people with dementia at the end of life which was subsequently shared with the group and further discussion on how to use this toolkit in practice. There are now further discussions exploring the skills and confidence of Admiral Nurses having challenging conversations at end of life, including Advance Care Planning, and how a practice development approach could assist development in this area.

Conclusion A practice development approach to Admiral Nurses learning on end of life care will ensure that their development is person centred, innovative and valuable. This model of supporting specialist nurses in their practice allows better understanding and awareness to collaborate with palliative care colleagues.

\section{P-103 A COMMUNITY OF PRACTICE: BUILDING CAPABILITY TO PROVIDE HIGH QUALITY DEMENTIA CARE}

${ }^{1}$ Marie Cooper, ${ }^{2}$ Karen Harrison Dening. ${ }^{1}$ Hospice UK, London, UK; ${ }^{2}$ Dementia UK, London, UK

10.1136/bmjspcare-2017-hospice.129

In recent years there has been a significant increase in policy and guidance, across many countries, to promote palliative and end-of-life care for non-malignant life-limiting conditions. Most notably for people with dementia (PWD) at the end-oflife and facilitate better access to palliative care. PWD, particularly those in the advanced stages, may experience poor endof-life care because they may not be perceived to have a terminal illness. There is concern in some settings and services that staff are ill - equipped to care for PWD, in respect of knowledge and skills.

Both Hospice UK and Dementia UK thus launched the 'Dementia in palliative and end-of-life care Community of Practice' to bring together practitioners keen, in the spirit of mutual learning, to share knowledge and practice and provide high quality palliative and end-of-life care for families affected by dementia. At the inaugural meeting a nominal group technique was used to identify learning needs across both groups of clinicians. Thirteen learning needs were identified, of which five were ranked as priorities to be met during future meetings. Shared learning through a community of practice is a way of harnessing the expertise across the two care domains, with the common objective of improving the lives, and deaths of PWD and in better support of their families. We hold two community meetings a year and due to increase in engagement will be hosting a conference in 2017. Events are well evaluated and membership is growing.

\section{P-104 DEMENTIA HUBS IN A HOSPICE}

1,2Penny Foulds, 1,2Vivienne Trott. 'Lancaster University, Division of Biomedical and Life Sciences, Faculty of Health and Medicine, Lancaster, UK; ${ }^{2}$ Trinity Hospice and Palliative Care Services, Blackpool, UK

10.1136/bmjspcare-2017-hospice. 130 